\title{
PRIAPISMO SECUNDARIO A REACCIÓN LEUCEMOIDE PARANEOPLÁSICA EN PACIENTE CON CÁNCER DE VEJIGA
}

\author{
A. RODRÍGUEZ ALONSO, E. ROMERO PICOS*, G. SUÁREZ PASCUAL, \\ C. BONELLI MARTÍN, A. GONZÁLEZ BLANCO, J. LORENZO FRANCO, \\ M.Á. CUERPO PÉREZ, J. NIETO GARCÍA
}

Servicio de Urología. *Servicio de Hematología. Hospital Arquitecto Marcide. El Ferrol. La Coruña.

Actas Urol Esp. 28 (7): 539-543, 2004

\section{RESUMEN}

PRIAPISMO SECUNDARIO A REACCIÓN LEUCEMOIDE PARANEOPLÁSICA EN PACIENTE CON CÁNCER DE VEJIGA

El priapismo asociado a tumores sólidos suele producirse por metástasis peneanas o por infiltración tumoral directa de los cuerpos cavernosos. Las neoplasias son responsables del 3-8\% de los casos de priapismo, y en el $80 \%$ de los casos el tumor primario es de origen génito-urinario.

La reacción leucemoide es un trastorno hematológico caracterizado por una leucocitosis superior a 50000/ $\mu 1$, que se desarrolla de forma reactiva ante diversos procesos patológicos como inflamaciones severas, infecciones diseminadas, intoxicaciones y tumores.

El mecanismo fisiopatológico que explica el priapismo secundario a reacción leucemoide es una obstrucción intravascular por hiperviscosidad sanguínea, que origina una estasis sanguínea en las venas cavernosas, impidiendo el drenaje de los cuerpos cavernosos.

Presentamos un caso de priapismo en un paciente con un cáncer de vejiga localmente avanzado, que se desencadenó por la hiperviscosidad sanguínea producida por una reacción leucemoide paraneoplásica.

PALABRAS CLAVE: Priapismo. Neoplasia vesical. Reacción leucemoide. Sindrome paraneoplásico. Hiperviscosidad sanguínea.

\section{ABSTRACT \\ PRIAPISM FOLLOWING PARANEOPLASTIC LEUKEMOID REACTION IN A PATIENT WITH BLADDER CANCER}

Priapism associated with solid tumours is usually produced by penile metastasis or direct tumour infiltration of the corpora cavernosa. Neoplasias are responsible for between 3-8\% of all cases of priapism, and in $80 \%$ of cases the primary tumour has a genito-urinary origin.

Leukemoid reaction is an haematological disorder characterised by a leukocytosis of over $50000 / \mu 1$, which develops reactively with different pathological processes such as severe inflammation, disseminated infections, intoxications and tumours.

The pathophysiologic mechanism that explains priapism following leukemoid reaction is an intravascular obstruction due to blood hyperviscosity, leading to a blood stasis in the cavernosa veins, and impeding the drainage of the corpora cavernosa.

We present a case of priapism in a patient with locally advanced bladder cancer, which was probably the result of blood hyperviscosity produced by a paraneoplastic leukemoid reaction.

KEY WORDS: Priapism. Bladder neoplasm. Leukemoid reaction. Paraneoplastic syndrome. Blood hyperviscosity. 
$\mathrm{E}^{\prime}$ término priapismo hace referencia a una erección prolongada, no relacionada con el deseo ni la estimulación sexuales ${ }^{1}$.

Desde el punto de vista etiológico, se pueden distinguir dos tipos de priapismo: priapismo idiopático y priapismo secundario. Los principales factores desencadenantes del priapismo secundario son las alteraciones hematológicas, las disfunciones neurógenas, las neoplasias, los trastornos metabólicos, las enfermedades infecciosas y la acción de diversas sustancias tóxicas y fármacos, entre los cuales cabe destacar los agentes vasoactivos intracavernosos utilizados para el tratamiento de la disfunción eréctil, que constituyen la principal causa de priapismo en la actualidad $^{2}$.

El síndrome paraneoplásico es un conjunto de manifestaciones clínicas sistémicas, que se presentan en pacientes con enfermedad neoplásica sin estar directamente causadas por invasión, compresión u obstrucción tumorales, y que evolucionan paralelamente a la neoplasia, remitiendo habitualmente tras el tratamiento efectivo de la misma ${ }^{3,4}$. Desde esta perspectiva, la aparición de una reacción leucemoide (RL) en el contexto de un cáncer, puede considerarse un sindrome paraneoplásico.

El desarrollo de RL en pacientes con neoplasia vesical es un hecho infrecuente, que se relaciona con la carga tumoral, se presenta habitualmente en fases tardias de la enfermedad y suele asociarse a un mal pronóstico ${ }^{5}$.

Presentamos un caso de priapismo en el contexto de un cáncer de vejiga, que se desencadenó por la hiperviscosidad sanguínea generada por una reacción leucemoide paraneoplásica, y no por metástasis peneanas como es habitual en este tipo de pacientes.

\section{CASO CLÍNICO}

Paciente varón de 77 años, con antecedentes de tuberculosis pulmonar antigua, reflujo gastroesofágico e hipertensión arterial. Resección transuretral (RTU) de neoplasia vesical en 2 ocasiones: T1G1 y T1G3. RTU de próstata: hipertrofia prostática benigna.

El paciente no acudió al seguimiento programado de su neoplasia vesical y en cistoscopia realizada dos años después de la última RTU, se detectaba recidiva tumoral sólida de gran tamaño, que afectaba a la cara lateral derecha, cara posterior, trígono, cuello vesical y uretra prostática. Posteriormente, y de forma urgente, el paciente ingresaba por macrohematuria anemizante.

Las pruebas de laboratorio al ingreso mostraban los siguientes datos: hemograma: 68000 leucocitos $/ \mu 1$ (93,4\% neutrófilos, 2,7\% linfocitos), hemoglobina 8,4 g/dl, plaquetas 225000/ $\mu 1$. Bioquímica sanguínea: fosfatasa alcalina $651 \mathrm{U} / 1$ 37ํㅡㄴ LDH 464 U/1 37ํ․ Coagulación: normal.

Durante el ingreso se realizaron hemogramas seriados, observando un incremento progresivo de la hiperleucocitosis, que alcanzaba 126600 leucocitos $/ \mu 1$ (97\% neutrófilos, 0\% linfocitos). Paralelamente se apreciaron datos de anemia recurrente, que precisó múltiples transfusiones de hematíes y plasma fresco.

El urocultivo y los hemocultivos eran negativos.

Se realizó frotis de sangre periférica, sin observar blastos, ni otro tipo de células que hicieran sospechar patología primaria de la médula ósea. Se efectuó igualmente tinción específica de fosfatasa alcalina leucocitaria (FAL), observando unos valores aumentados en dos determinaciones diferentes (144 y 170).

Ante la ausencia de fiebre, la negatividad persistente de los cultivos, los hallazgos del frotis de sangre periférica, y la elevación de los valores de FAL, se realizó el diagnóstico de reacción leucemoide paraneoplásica.

Se efectuó TAC abdómino-pélvico, en el que se evidenciaba una gran masa vesical irregular, de localización preferentemente derecha, que ocasionaba uropatía obstructiva ipsilateral, infiltrando el plano graso perivesical, el lóbulo prostático derecho y ambas vesículas seminales. Se observaban asimismo, adenopatías iliacas externas, obturatrices y femorales izquierdas (la mayor de $3,5 \mathrm{~cm})$. Dichos hallazgos eran compatibles con neoplasia vesical localmente avanzada T4a N2 MX. No se objetivaban metástasis en cuerpos cavernosos (Figs. 1 y 2).

Se efectuó asimismo una gammagrafía ósea (99m-Tc MDP), en la que no se observaban depósitos patológicos del trazador.

Se realizó a continuación, RTU hemostática paliativa de la tumoración, observando afectación 


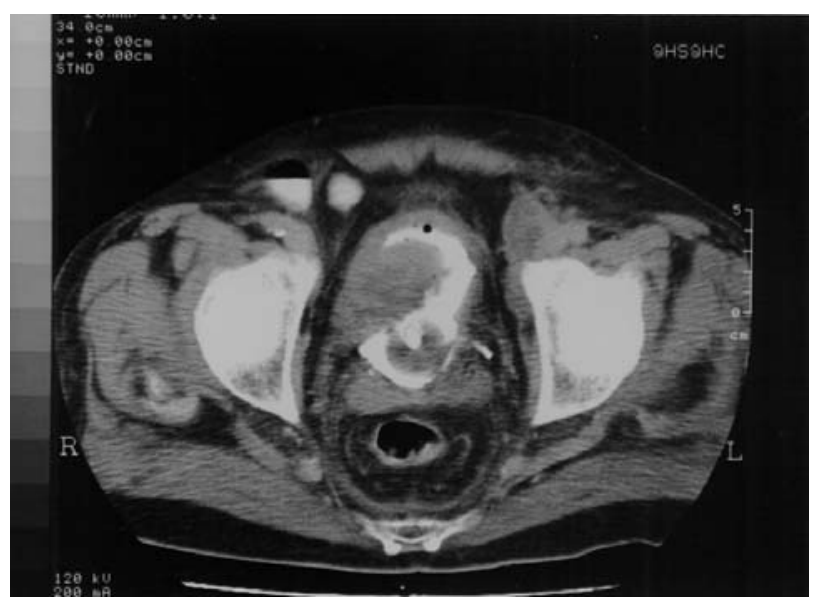

FIGURA 1. TAC pélvico en el que se advierte una gran tumoración vesical, con extensión perivesical y adenopatias femorales izquierdas.

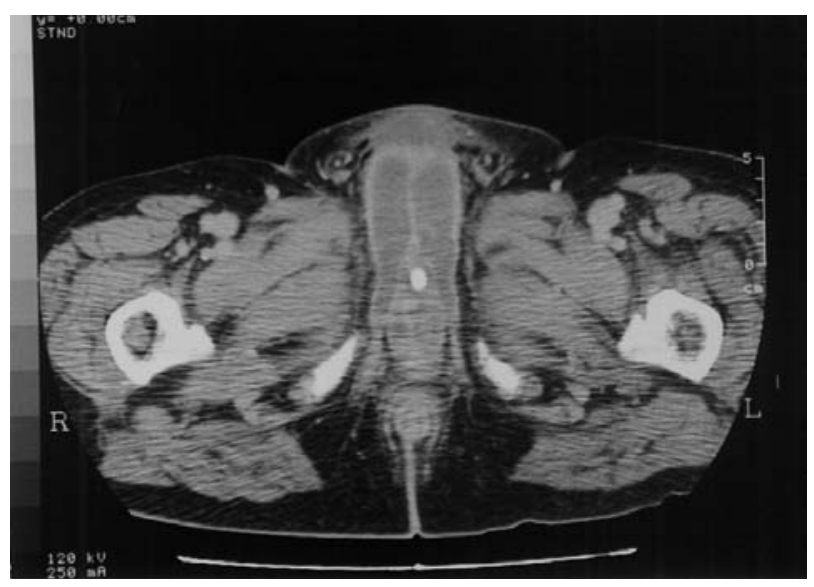

FIGURA 2. TAC pélvico en el que se observa la raiz de ambos cuerpos cavernosos, sin evidenciarse afectación tumoral de los mismos.

macroscópica de la grasa perivesical y del parénquima prostático. El estudio anatomopatológico del material de resección reveló la existencia de un carcinoma de células transicionales de vejiga pobremente diferenciado con infiltración de grasa perivesical y parénquima prostático (T4aG3).

El quinto día post-operatorio, el paciente refería erección y dolor peneano intenso, de instauración progresiva en el transcurso de aproximadamente 72 horas. A la exploración física se observaba priapismo de ambos cuerpos cavernosos, sin evidencia de nódulos peneanos palpables (Fig. 3).

El priapismo fue inicialmente tratado mediante drenaje de ambos cuerpos cavernosos con aguja de 14G, obteniendo una sangre oscura y

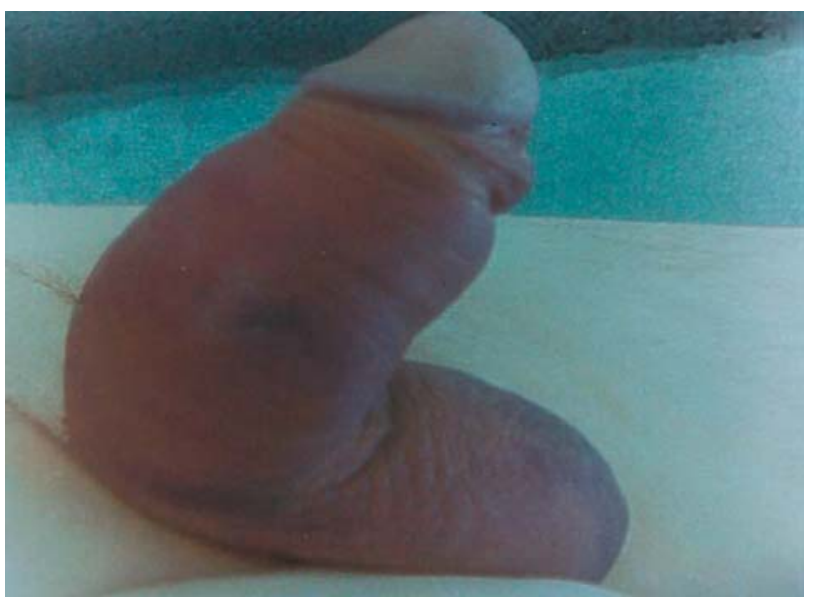

FIGURA 3. Priapismo refractario al tratamiento médico con agentes alfa-adrenérgicos.

viscosa, cuya gasometría $(\mathrm{pH}=6,95 ; \mathrm{pO} 2=45$ $\mathrm{mmHg}$ ) era compatible con priapismo veno-oclusivo. Esta maniobra se efectuó en varias ocasiones sin lograr la resolución del priapismo. A continuación se realizó lavado de cuerpos cavernosos con suero fisiológico, para proceder a continuación a la irrigación de los mismos con una solución de fenilefrina ( $5 \mathrm{mg}$ de fenilefrina en 500 $\mathrm{ml}$ de suero fisiológico). Ninguna de estas maniobras resultó efectiva, por lo que se decidió realizar tratamiento quirúrgico.

Se practicó un shunt distal córporo-glandular según técnica de Winter, logrando una detumescencia parcial (Fig. 4). 24 horas después de la realización del shunt el paciente presentaba recidiva del priapismo, realizándose entonces un

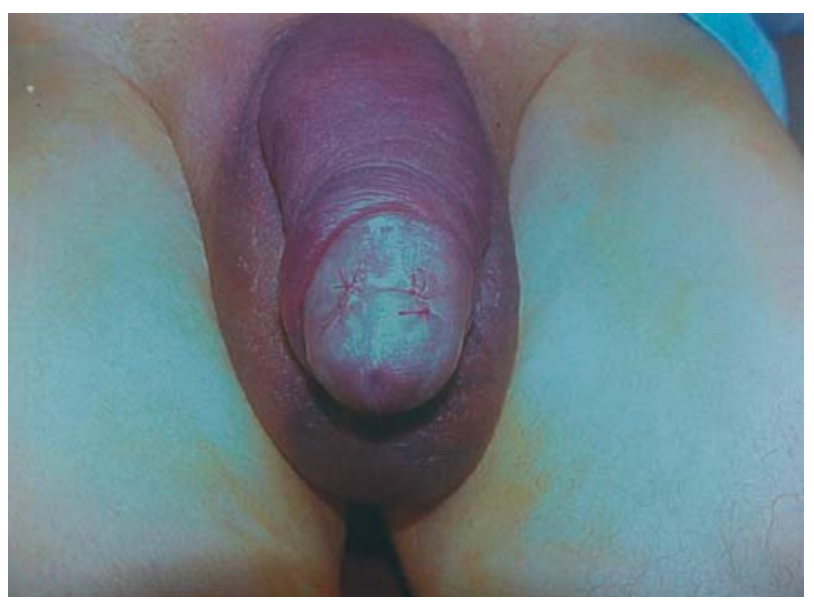

FIGURA 4. Detumescencia peneana parcial tras realización de shunt distal córporo-glandular de Winter. 
legrado de ambos cuerpos cavernosos a través de los orificios balánicos de la fístula. La detumescencia obtenida con esta maniobra fue considerablemente menor que con el primer procedimiento, recidivando el priapismo pocas horas después.

La citología aspirativa de ambos cuerpos cavernosos, efectuada durante el primer procedimiento quirúrgico, era negativa para carcinoma de células transicionales.

Debido a la imposibilidad de tratar de forma eficaz la enfermedad de base del paciente y al estado terminal del mismo, se decidió realizar exclusivamente tratamiento sintomático paliativo. El paciente es exitus 10 días después de la realización del legrado de cuerpos cavernosos.

\section{DISCUSIÓN}

La RL es un trastorno hematológico que analíticamente simula una leucemia mieloide crónica (LMC). Se caracteriza por leucocitosis intensa, que por definición ha de ser superior a $50000 / \mu \mathrm{l}$, en la RL neutrofilica, pudiendo alcanzarse cifras superiores a $200000 / \mu 1^{3,6}$.

Son diversos los procesos patológicos que subyacen en una RL, siendo los más frecuentes las inflamaciones severas, las infecciones diseminadas, las intoxicaciones y los tumores. El efecto estimulador sobre la médula ósea es el nexo común de todos estos procesos ${ }^{3,6,7}$.

La RL paraneoplásica es habitualmente mieloide y son los cánceres de pulmón, estómago, páncreas, mama y tiroides los que más frecuentemente las ocasionan ${ }^{3,6}$. En las neoplasias génito-urinarias, la RL y los síndromes paraneoplásicos en general, se observan con poca frecuencia, siendo la excepción el adenocarcinoma renal $^{3}$.

Se desconoce el mecanismo exacto por el cual una neoplasia puede producir una estimulación de la médula ósea, originando una $\mathrm{RL}^{4,6,7}$. Uno de los mecanismos fisiopatológicos estudiados es la liberación autónoma, por parte de las células tumorales, de ciertas citoquinas, o de factores estimulantes de la hematopoyesis, como el factor estimulante de las colonias de granulocitos (G$\mathrm{CSF})^{6-8}$. Otros posibles mecanismos causales de RL paraneoplásica son la necrosis tumoral y la irritación directa de la médula ósea ${ }^{3,7}$.
El diagnóstico diferencial de la RL debe realizarse inevitablemente con la LMC. Entre ambas entidades existe una clara diferencia conceptual, ya que mientras la RL es un cuadro reactivo a infecciones, tumores, etc., la LMC es una enfermedad clonal de la médula ósea.

La RL cursa con una serie blanca aumentada en número, pero sin alteraciones dishematopoyéticas; presencia de gránulos citoplasmáticos intensamente teñidos (granulación tóxica); ausencia de blastos en el frotis de sangre periférica; bajo recuento de basófilos; ausencia de cromosoma Philadelphia y unos niveles elevados de fosfatasa alcalina leucocitaria, como se pudo evidenciar en el paciente de nuestro caso, cuyos valores de FAL fueron 144 y 170, en dos diferentes determinaciones ${ }^{6,7}$.

En la LMC, por el contrario, se observa una serie blanca patológica, tanto cuantitativa como cualitativamente; ausencia de granulación tóxica; presencia de blastos en sangre periférica; frecuente elevación de la cifra de basófilos; presencia de cromosoma Philadelphia y un valor de FAL de cero, o próximo a cero ${ }^{6,7}$.

El diagnóstico de RL en un paciente con neoplasia vesical se basa en la existencia de datos hematimétricos compatibles y de una cifra de FAL aumentada, no siendo necesario realizar biopsia de médula ósea en la mayoría de los casos.

El priapismo asociado a tumores sólidos suele producirse por metástasis peneanas o por infiltración tumoral directa de los cuerpos cavernosos. Las neoplasias son responsables del 3-8\% de los casos de priapismo ${ }^{2}$, y en el $80 \%$ de los casos el tumor primario es de origen génito-urinario ${ }^{9,10}$.

Los mecanismos que se han postulado para explicar la fisiopatología del priapismo asociado a neoplasias malignas son los siguientes: infiltración tumoral del cuerpo cavernoso; infiltración del sistema de drenaje venoso, con desarrollo de trombosis venosa; e infiltración y/o irritación de la inervación erectógena, sin afectación directa del cuerpo cavernoso ${ }^{9}$.

Ninguna de las posibilidades anteriormente descritas se ajusta a nuestro caso, ya que la exploración física y los estudios de imagen no evidenciaron la existencia de metástasis peneanas, y el priapismo por irritación neural es una teoría muy difícil de demostrar. 
Desde nuestro punto de vista, la hiperviscosidad sanguínea ocasionada por la hiperleucocitosis es el único factor demostrable en nuestro paciente, con capacidad para desencadenar un priapismo.

El mecanismo fisiopatológico que explica este tipo de priapismo es una obstrucción mecánica intravascular secundaria a hiperviscosidad sanguínea. Esta hiperviscosidad origina una estasis sanguínea de las venas cavernosas, que impide el drenaje de los cuerpos cavernosos y desencadena un priapismo ${ }^{11}$.

Las técnicas radiológicas habitualmente utilizadas para demostrar la afectación tumoral de los cuerpos cavernosos son TAC, RM y cavernosografía ${ }^{9}$. El diagnóstico anatomopatológico puede efectuarse bien mediante citología aspirativa de cuerpos cavernosos o bien mediante biopsia de lesiones peneanas clínicamente demostrables $^{9,12}$. En el paciente de nuestro caso, la citología aspirativa de ambos cuerpos cavernosos no detectó células neoplásicas, lo cual refrenda los hallazgos de la exploración física y radiológica.

El tratamiento del priapismo debe estar orientado a resolver la etiología del mismo, intentando interrumpir la estimulación patológica desencadenante, siempre que esto sea posible ${ }^{2}$. Como se ha comentado con anterioridad, la evolución de los sindromes paraneoplásicos es paralela a las neoplasias, de forma que el tratamiento efectivo de la neoplasia vesical sería el único modo de lograr la remisión de la reacción leucemoide y de disminuir la hiperviscosidad sanguínea que dio lugar al priapismo.

La irrigación intracavernosa con agentes alfaadrenérgicos, la realización de fístulas córporoglandulares o el legrado de los cuerpos cavernosos, son medidas terapéuticas de eficacia transitoria, si no se elimina el trastorno subyacente, como se pudo comprobar en el paciente de nuestro caso.

Algunos autores indican penectomía total en casos de priapismo secundario a metástasis peneana, cuando el estado general del paciente es bueno y no existen metástasis en otras localizaciones $^{10,12}$. La realización de un tratamiento agresivo es, desde nuestro punto de vista, desaconsejable en pacientes con una baja probabilidad de supervivencia a corto plazo.

\section{REFERENCIAS}

1. KEOGHANE SR, SULLIVAN ME, MILLER MAW.: The aetiology, patogénesis and management of priapism. BJU Int 2002; 90: 149-154.

2. ARRONDO JL, NAPAL S.: Priapismo. En: Pomerol JM, Arrondo JL, editores. Práctica Andrológica. 1a ed. Barcelona: Masson-Salvat Medicina 1994: 737-747.

3. REIG RUIZ C, VALLEJO GIL C, MOROTE ROBLES J, SOLER ROSELLÓ A.: Reacción leucemoide y trombocitosis en sarcoma de vejiga. Actas Urol Esp 1994; 18 (6): 687-689.

4. PÉREZ ARBEJ JA, NOGUERAS GIMENO MA, MARTÍNEZ PÉREZ E et al.: Fiebre y reacción leucemoide en cáncer vesical: a propósito de un caso. Arch Esp Urol 1991; 44 (3): 309-312.

5. PASCUAL SAMANIEGO M, TORRECILLA GARCÍARIPOLL JR, CALLEJA ESCUDERO J, EGEA CAMACHO J, RIVERA FERRO J, FERNÁNDEZ DEL BUSTO E.: Hipercalcemia, reacción leucemoide y trombocitosis como presentación paraneoplásica del carcinoma renal de células transicionales. Actas Urol Esp 2001; 25 (5): 400-405.

6. MILLER JI, SARVER RG, DRACH GW.: Leukemoid reaction: a rare paraneoplastic syndrome associated with advanced bladder carcinoma. Urology 1994; 44 (3): 444-446.

7. PERTUSA PEÑA C, LLARENA IBARGUREN R, ZABALA JA, ARRUZA ECHEVARRÍA A, ARREGUI ERBINA P.: Reacción leucemoide en el carcinoma vesical. Arch Esp Urol 1988; 41 (3): 231-232.

8. WETZLER M, ESTROV Z, TALPAZ M, MARKOWITZ A, GUTTERMAN JU, KURZROCK R.: Granulocytemacrophage colony-stimulating factor as a cause of paraneoplastic leukaemoid reaction in advanced transitional cell carcinoma. J Intern Med 1993; 234 (4): 417-420.

9. CHAN PTK, BÉGIN LR, ARNOLD D, JACOBSON SA, CORCOS J, BROCK GB.: Priapism secondary to penile metastasis: a report of two cases and a review of the literature. J Surg Oncol 1998; 68: 51-59.

10. GRANADOS LOARCA EA, FARIÑA PÉREZ L, HOCSMAN BARTFELD H, SANTAULARIA SEGURA JM: Priapismo maligno. Legrado de los cuerpos cavernosos como medida paliativa. Arch Esp Urol 1991; 44 (2): 203-204.

11. SÁENZ DE TEJADA I.: Priapismo: fisiopatología y tratamiento médico. En: Sáenz de Tejada I, Allona Almagro A, editores. Erección, eyaculación y sus trastornos. $1^{\underline{a}}$ ed. Madrid: Fomento Salud 1997: 257-273.

12. MURO BIDAURRE I, AZPIAZU ARNAIZ P, RECARTE BARRIOLA JA, HERNAEZ MANRIQUE I.: Carcinoma metastático de pene: evaluación clínica, tratamiento y revisión de la literatura. Arch Esp Urol 1999; 52 (9): 994-998.

Dr. A. Rodríguez Alonso

(Servicio de Urología)

Hospital Arquitecto Marcide

Ctra. San Pedro de Leixa, s/n

15405 El Ferrol (La Coruña)

(Trabajo recibido el 19 septiembre de 2003) 